\title{
Change of Serum BNP Between Admission and Discharge After Acute Decompensated Heart Failure Is a Better Predictor of 6-Month All-Cause Mortality Than the Single BNP Value Determined at Admission
}

\author{
Renato De Vecchis ${ }^{\mathrm{a}, \mathrm{f}}$, Carmelina Ariano ${ }^{\mathrm{a}}$, , Giuseppe Giandomenico ${ }^{\mathrm{c}}$, Marco Di Maio ${ }^{\mathrm{d}}$, Cesare Baldi
}

\begin{abstract}
Background: B-type natriuretic peptide (BNP) is regarded as a reliable predictor of outcome in patients with acute decompensated heart failure (ADHF). However, according to some scholars, a single isolated measurement of serum BNP at the time of hospital admission would not be sufficient to provide reliable prognostic information.

Methods: A retrospective study was carried out on patients hospitalized for ADHF, who had then undergone follow-up of at least 6 months, in order to see if there was any difference in midterm mortality among patients with rising BNP at discharge as compared to those with decreasing BNP at discharge. Medical records had to be carefully examined to divide the case records into two groups, the former characterized by an increase in BNP during hospitalization, and the latter showing a decrease in BNP from the time of admission to the time of discharge.
\end{abstract}

Results: Ultimately, 177 patients were enrolled in a retrospective study. Among them, 53 patients (29.94\%) had increased BNPs at the time of discharge relative to admission, whereas 124 (70.06\%) exhibited decreases in serum BNP during their hospital stay. The group with patients who exhibited BNP increases at the time of discharge had higher degree of congestion evident in the higher frequency of persistent jugular venous distention (odds ratio: 3.72; $\mathrm{P}=0.0001$ ) and persistent orthopnea at discharge (odds ratio: 2.93; $\mathrm{P}=0.0016$ ). Moreover, patients with increased BNP at the time of discharge had

Manuscript accepted for publication August 17, 2016

aCardiology Unit, Presidio Sanitario Intermedio "Elena d'Aosta", ASL Napoli 1 Centro, Napoli, Italy

bDivision of Cardiology, Casa di Cura "Sollievo della Sofferenza", S. Giovanni Rotondo, Italy

cHospital Directorate, Presidio Sanitario Intermedio "Elena d'Aosta", ASL Napoli 1 Centro, Napoli, Italy

${ }^{\mathrm{d}}$ Department of Cardiology, Second University of Napoli, Monaldi Hospital, Napoli, Italy

eHeart Department, Interventional Cardiology, A.O.U. "San Giovanni di Dio e Ruggi d'Aragona", Salerno, Italy

fCorresponding Author: Renato De Vecchis, Cardiology Unit, Presidio Sanitario Intermedio "Elena d'Aosta", ASL Napoli 1 Centro, Napoli, Italy.

Email: devecchis.erre@virgilio.it

doi: http://dx.doi.org/10.14740/jocmr2691w a lower reduction in inferior vena cava maximum diameter $(1.58 \pm$ $2.2 \mathrm{~mm}$ vs. $6.32 \pm 1.82 \mathrm{~mm}$; $\mathrm{P}=0.001$ (one-way ANOVA)). In contrast, there was no significant difference in weight loss when patients with increased BNP at discharge were compared to those with no such increase. A total of 14 patients (7.9\%) died during the 6-month follow-up period. Cox proportional hazard analysis revealed that BNP increase at the time of discharge was an independent predictor of 6-month all-cause mortality after adjustment for age, sodium at discharge, creatinine at discharge and New York Heart Association (NYHA) class at discharge (hazard ratio 34.49 ; $95 \%$ confidence intervals: $4.55-261.06 ; \mathrm{P}=0.001)$.

Conclusions: Among patients with recent ADHF, increased BNP at the time of discharge from the hospital entailed a higher grade of congestion and higher 6-month mortality.

Keywords: B-type natriuretic peptide; Heart failure; Congestion

\section{Introduction}

Prognostic studies have shown that serum B-type natriuretic peptide (BNP) values, measured after treatment were more predictive of post-discharge mortality and cardiovascular events, compared to the values recorded at the time of admission [1]. Moreover, Cohen-Solal and colleagues found that patients with a fall of the BNP serum level, especially when occurring on the fifth day after onset of therapy, had a reduced risk of mortality in despite of a BNP value detected at admission significantly higher than that found in the other patients [2]. Increased BNP in hospital is sometimes detected, by comparing values found at admission with those seen at discharge, despite the appropriate treatment of acute HF, suggesting that there may be other determinants able to influence the level of serum BNP [3] in addition to the decisive main factor that is the grade of hemodynamic overload of the ventricular myocardium.

\section{Aims}

In this study, we aimed to detect the characteristics and out- 


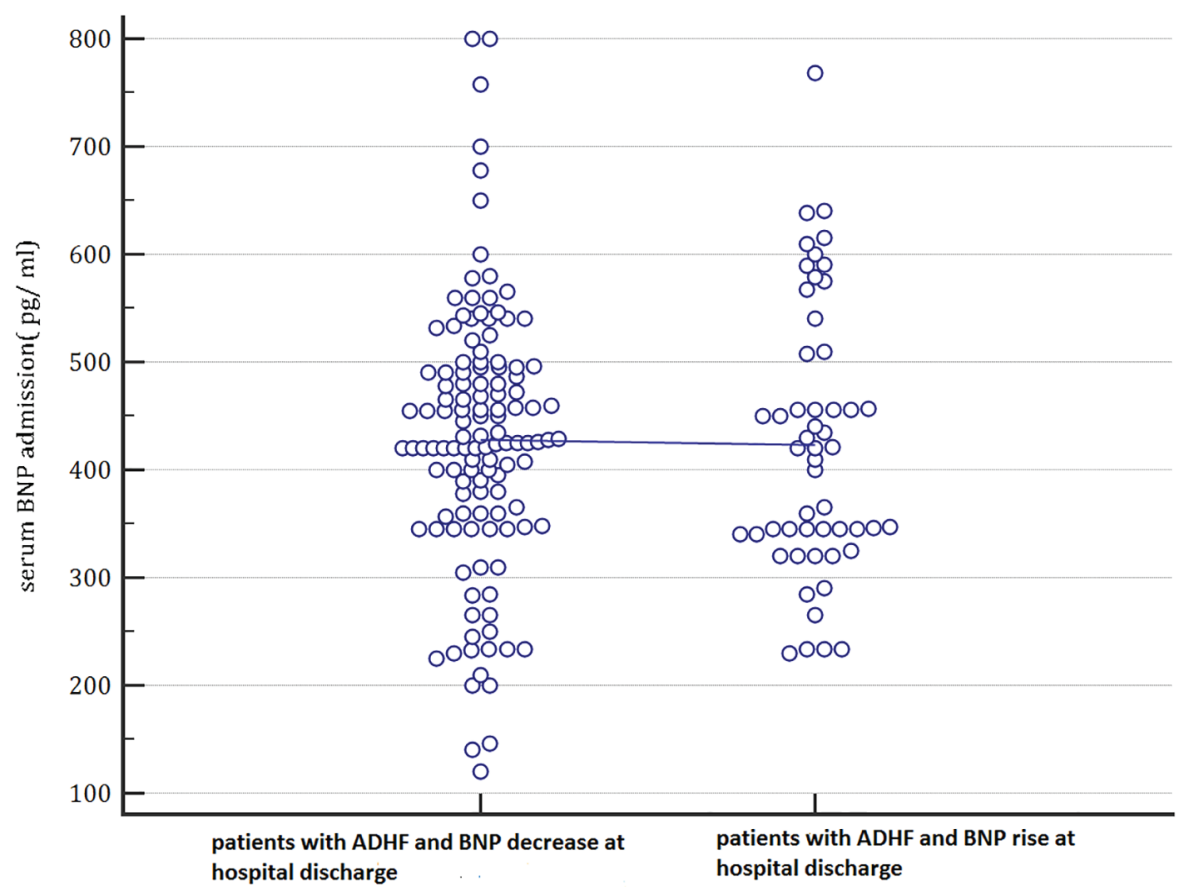

Figure 1. In this plot, the admission serum BNP values are categorized depending on the values that they will assume at the hospital discharge (patients with BNP decrease during hospital stay until discharge compared to patients with BNP rising at hospital discharge). Based on these findings, admission serum BNP was not able to predict the subsequent evolution of BNP levels. Indeed, there were not any differences between basal BNP mean values of patients who will evolve into a BNP decrease at hospital discharge and of those who will show a BNP rise at hospital discharge. ADHF: acute decompensated heart failure; BNP: B-type natriuretic peptide; pg: pictograms.

comes of patients hospitalized for acute decompensated heart failure (ADHF), all characterized by values of BNP at discharge that were higher than those found at admission. This group of patients with BNP rising at discharge was compared with other patients similarly affected by ADHF who instead had exhibited a BNP reduction at the end of hospital stay compared to the admission values. Other study objectives included determining the characteristics and grade of congestion of patients with BNP increase at the time of discharge in comparison with those who had a reduction of discharge BNP.

\section{Methods}

In this retrospective study, all data were collected from paper or electronic medical records related to the activities of hospitalization and subsequent follow-up of patients with confirmed diagnosis of ADHF who belonged to the Division of Cardiology of the Clinic "Sollievo della Sofferenza" of San Giovanni Rotondo (Italy) during the period from January 2012 to January 2015. For inclusion in our retrospective study, the patients were required to have received a diagnosis of ADHF entailing hospitalization. Moreover, both the BNP value measured at the time of hospital admission as well as the value of BNP corresponding to the time of discharge should have been reported in the medical sheets in order to comply with the aims of the study. Finally, for each patient included in our retrospective study, availability of clinical follow-up data concerning the first 6 months after discharge was required. Pertaining data were collected with the consent of the Hospital Directorate; they derived from careful evaluation of clinical records, in strict accordance with the rules and regulations that apply to the patient's privacy preservation.

In this retrospective study, our primary endpoint was 6-month all-cause mortality. Among the signs deduced from physical examination, we used jugular venous distention (JVD) and orthopnea for assessing and grading volume status, according to other authors $[4,5]$. In addition, we used further two objective variables, recognized suitable to evaluate decongestion [6-8]: weight loss and reduction in the inferior vena cava (IVC) maximum (i.e., expiratory) diameter from admission to discharge.

\section{Statistical analysis}

Patients with or without BNP increase at discharge were compared as regards their main signs and symptoms of clinical congestion as well as with respect to the mortality at 6 months. Continuous variables were expressed as mean \pm standard deviation and were tested for normality of distribution using the D'Agostino-Pearson test. They were compared using one-way analysis of variance (ANOVA) and/or independent samples $t$-test for normally distributed variables, or using Mann-Whitney $U$ test for non-normally distributed 
Table 1. In Patients Hospitalized for Acute Decompensated Heart Failure, the Odds of Persistent Jugular Venous Distention Is Significantly Higher Among Patients With BNP Rising at Discharge (Yes) Compared to Those Free From This Laboratory Finding (No)

\begin{tabular}{|c|c|c|c|}
\hline & \multicolumn{3}{|c|}{ JVD } \\
\hline & JVD persistence & JVD regression & Total \\
\hline \multicolumn{4}{|c|}{$\mathrm{BNP}$ rising at discharge } \\
\hline Yes & 32 & 21 & 53 \\
\hline No & 36 & 88 & 124 \\
\hline Total & 68 & 109 & 177 \\
\hline Odds ratio & 3.7249 & & \\
\hline $95 \% \mathrm{CI}$ & $1.8997-7.3034$ & & \\
\hline z statistic & 3.828 & & \\
\hline Significance level & $\mathrm{P}=0.0001$ & & \\
\hline
\end{tabular}

For further explanations, please see the text. BNP: B-type natriuretic peptide; JVD: jugular venous distention.

variables. Paired sample $t$-test was used to compare the grade of congestion within each group on admission and discharge. Categorical variables were described as counts and percentages and compared using the Chi-square test. Multivariate Cox proportional hazards regression was used to ascertain whether BNP increase at discharge was an independent predictor of 6-month all-cause mortality. The variables used in this model were those that achieved statistical significance at $\mathrm{P}<0.05$ upon univariate analysis or those known to be a post-discharge mortality predictor based on prior studies. All statistical tests were performed with a commercially available statistical analysis program (SPSS 15.0 for Windows, SPSS Inc., Chicago, IL, USA). All statistical significance was assessed using a two-sided P values. A P-value less than 0.05 was considered statistically significant.

\section{Results}

\section{Patient characteristics}

A total of 177 patients (mean age 74 years, 75\% males) admitted with ADHF who had their BNP checked on admission and discharge were included in our analysis. These cases were divided into two groups for comparison based on whether they had BNP increase at discharge relative to admission (53 patients, $29.94 \%$ ) or not (124 patients, $70.06 \%)$. There was not any significant difference between both groups with regard to admission BNP (423.22 \pm 124.286 pg/mL vs. $427.84 \pm 123.22$ $\mathrm{pg} / \mathrm{mL}$, in patients with and without BNP increase at discharge, respectively, $\mathrm{P}=0.820$ ) (Fig. 1). Conversely, discharge BNP was significantly higher in patients with BNP rise compared to those without an increase in BNP at discharge (591.47 \pm $213.81 \mathrm{pg} / \mathrm{mL}$ vs. $170.31 \pm 90.10 \mathrm{pg} / \mathrm{mL}$, respectively; $\mathrm{P}<$ $0.001)$. Most patients $(86 \%)$ were in New York Heart Association (NYHA) class IV at admission.

\section{Clinical and objective markers of congestion}

By physical exam, patients with BNP increase had higher degree of congestion evident in the higher frequency of patients who had persistence of JVD at discharge (60.3\% vs. $29.03 \%$, odds ratio: $3.72,95 \%$ confidence interval (CI): 1.8997 - 7.3034; $\mathrm{P}=0.0001$ ) (Table 1) as well as persistence of orthopnea at discharge (64.1\% vs. $37.9 \%$, odds ratio: $2.9317,95 \%$ CI: 1.5025 - 5.7203, $\mathrm{P}=0.0016$ ) (Table 2) compared with patients with admission-to-discharge BNP reduction. With regard to objective markers of congestion, patients with BNP increase at the time of discharge had a lower reduction in IVC diameter from admission to discharge $(1.58 \pm 2.2 \mathrm{~mm}$ vs. $6.32 \pm 1.82 \mathrm{~mm}$, $\mathrm{P}=0.001$ ) (Fig. 2). By contrast, there was not any significant difference in weight loss by comparing patients characterized by BNP increase at discharge with those not involved by BNP rise. Indeed, in the former the weight loss was equal to 2.1308 $\pm 2.5133 \mathrm{~kg}$, whereas in the latter it was calculated equal to $2.50 \pm 1.8921 \mathrm{~kg}(\mathrm{P}=0.279$, one-way ANOVA $)$.

\section{Six-month mortality}

A total of 14/177 (7.9\%) patients died during a follow-up of 6 months. On multivariate Cox proportional hazards regression patients with BNP increase at discharge were more likely to die (hazard ratio: $34.49,95 \%$ CI: $4.55-261.06$; $\mathrm{P}=0.001$ ) compared with those who had BNP decrease at discharge relative to admission. Cox proportional hazard analysis revealed that BNP increase at the time of discharge in patients with previous ADHF was an independent predictor of 6-month all-cause mortality after adjustment for age, sodium at discharge, creatinine at discharge and NYHA class at discharge, which are known predictors of post-discharge mortality in HF, based on prior studies [9, 10]. Figure 3 is a forest plot of variables included in the multivariate model. In our regression model, also serum 
Table 2. In Patients Hospitalized for Acute Decompensated Heart Failure, the Odds of Persistent Orthopnea Is Significantly Higher Among Patients With BNP Rising at Discharge (Yes) Compared to Those Free From This Laboratory Finding (No)

\begin{tabular}{|c|c|c|c|}
\hline & & Orthopnea & \\
\hline & Persistence of orthopnea & Regression of orthopnea & Total \\
\hline $\mathrm{BNP}$ rising at disch & & & \\
\hline Yes & 34 & 19 & 53 \\
\hline No & 47 & 77 & 124 \\
\hline Total & 81 & 96 & 177 \\
\hline Odds ratio & 2.9317 & & \\
\hline $95 \% \mathrm{CI}$ & $1.5025-5.7203$ & & \\
\hline z statistic & 3.154 & & \\
\hline Significance level & $\mathrm{P}=0.0016$ & & \\
\hline
\end{tabular}

For further explanations, please see the text. BNP: B-type natriuretic peptide.

BNP at admission was included, considering that this factor was regarded as a reliable predictor of all-cause mortality in the mid-term follow-up by other studies $[11,12]$. Nevertheless, in our population of patients with recent episode of $\mathrm{ADHF}$, serum BNP concentration measured at admission was not associated with increased risk of death during a 6-month follow-up.

\section{Discussion}

Based on our retrospective study conducted using various tools for statistical analysis (contingency tables, one-way ANOVA, multivariate Cox proportional hazards regression, etc.), we found that patients with recent ADHF, who also showed increased serum BNP at discharge, had a grade of decongestion significantly lower, either when clinically identified by observing the regression of JVD and orthopnea resolution, or when objectively detected through longitudinal, i.e., from admission-to-discharge, assessment of weight loss and reduction in maximum (expiratory) IVC diameter. BNP increase at the time of discharge was also independently associated with 6-month mortality after adjustment for known post-discharge

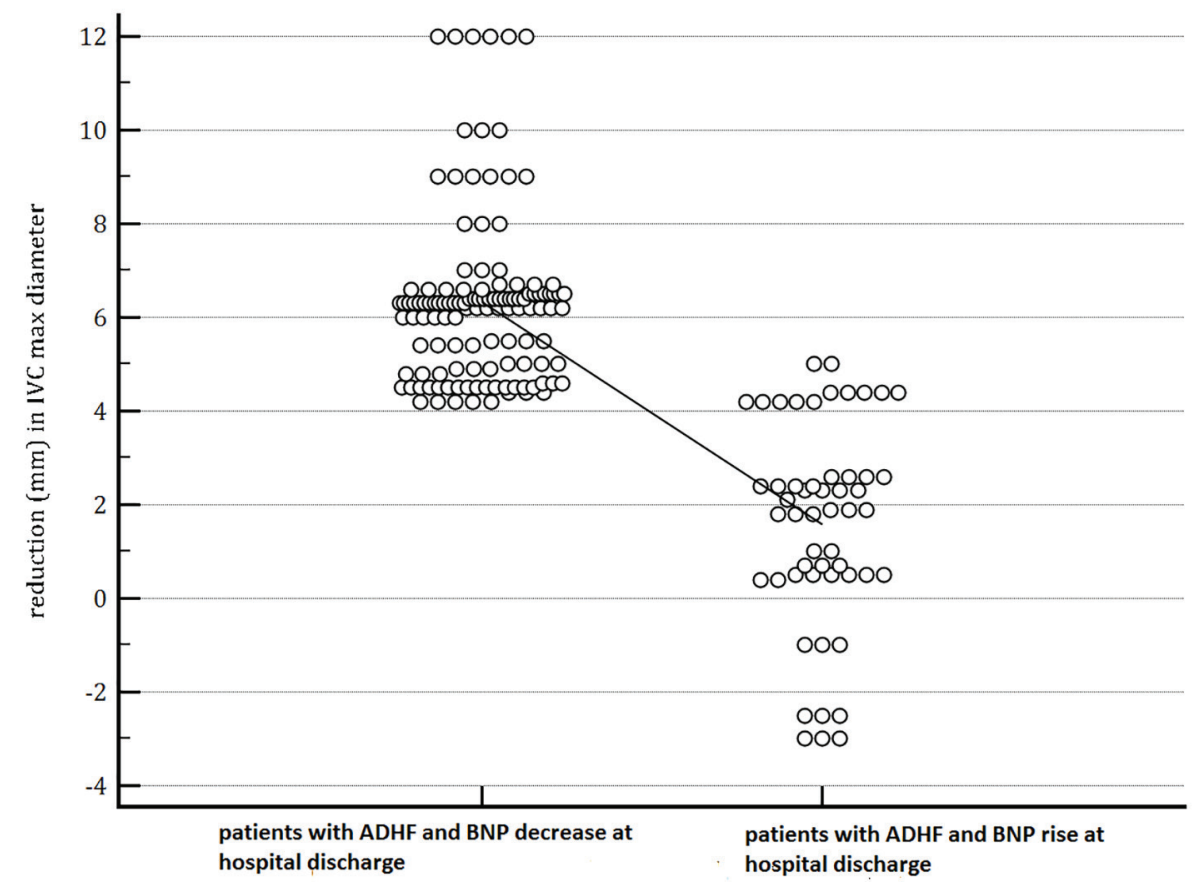

Figure 2. The figure shows that patients with BNP increase at the time of discharge had a lower reduction in IVC diameter from admission to discharge $(1.58 \pm 2.2 \mathrm{~mm}$ vs. $6.32 \pm 1.82 \mathrm{~mm}, \mathrm{P}=0.001)$. In the dot-plot, a continuous line connects the means of the two groups (patients with decreasing BNP and the patients whose BNP shows an increase at discharge). 


\begin{tabular}{lrcc} 
Covariates & \multicolumn{3}{c}{ Statistics for each covariat } \\
& $\begin{array}{c}\text { Hazard } \\
\text { ratio }\end{array}$ & $\begin{array}{c}\text { Lower } \\
\text { limit }\end{array}$ & $\begin{array}{c}\text { Upper } \\
\text { limit }\end{array}$ \\
Creatinine at discharge & 4.146 & 2.570 & 6.686 \\
Serum Na+ (mEq/l )at discharge & 0.749 & 0.665 & 0.845 \\
NYHA class at discharge & 5.793 & 2.841 & 11.814 \\
Age & 1.056 & 0.948 & 1.176 \\
Serum BNP at admission $(\mathrm{pg} / \mathrm{ml})$ & 1.003 & 0.999 & 1.007 \\
BNP rise ( $\mathrm{pg} / \mathrm{ml})$ at discharge & 34.492 & 4.557 & 261.066
\end{tabular}

p-Value
$<0.001$
$<0.001$
$<0.001$
0.324
0.213
0.001

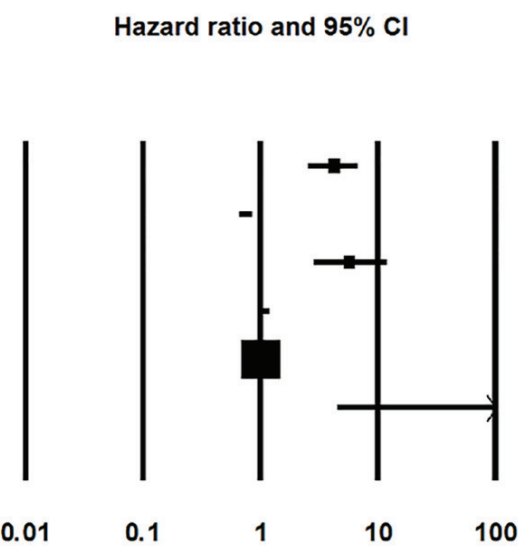

Lower mortality Higher mortality

Hazard Ratios and 95\% Confidence Intervals

Figure 3. Forest plot summarizing results of Cox proportional hazard analysis of predictors of 6-month all-cause mortality in our retrospective study that analyzed 177 patients hospitalized for acute decompensated heart failure and undergone clinical followup after their hospital discharge. BNP: B-type natriuretic peptide; NYHA: New York Heart Association.

mortality predictors in HF patients $[9,10]$ including age, discharge creatinine, discharge sodium, and discharge NYHA class. We suspect that the higher mortality in this group may be attributed to the lower grade of decongestion whether due to inefficient diuresis, vasodilation and renin-angiotensin-aldosterone system inhibition or, more importantly, due to worse underlying HF pathology, compared with those with admission-to-discharge BNP reduction. Indeed, serum BNP values at admission were not significantly different in the group of HF patients $(n=53)$, who subsequently developed an increase in $\mathrm{BNP}$ at discharge compared to that comprising the HF patients $(\mathrm{n}=124)$, who instead showed decreasing BNP at discharge. Moreover, using multivariate Cox proportional hazards regression, the variable "serum BNP concentration at admission" proved not to be associated to increased risk of death during a 6-month follow-up.

Thus, judging by our findings, higher all-cause mortality over a 6-month follow-up in HF patients with BNP increase at the time of discharge suggests that admission-to-discharge $\mathrm{BNP}$ change is superior to baseline absolute BNP value in predicting post-discharge outcomes.

The control of BNP secretion is not based solely on mechanisms of hemodynamic signage that come into play when cardiac intra-ventricular pressure exceeds a certain limit [3]. Indeed, it is likely that elevated levels of BNP at the time of admission to the hospital may arise from non-hemodynamic factors that have been shown to interfere with the secretion of BNP. For example, a high level of circulating norepinephrine or the coexistence of an altered renal function can affect serum BNP concentrations, pushing them upwards, in addition to the main determinant that is the degree of wall stress of the ventricular chambers $[3,13]$.

The difficulties related to the interpretation of numerous factors affecting the BNP test limit its role in day-to-day monitoring to guide therapy in acute HF [14]. Accordingly, the val- ue of serial BNP measurements in guiding therapy for patients with HF is not well established and was not recommended by societal guidelines [15]. Nonetheless, our findings still suggest a value for admission and discharge BNP measurements in acute $\mathrm{HF}$, as a BNP increase at discharge is an ominous prognostic factor associated with worse post-discharge outcomes which may have been driven by a higher degree of congestion related to less efficient diuresis or worse HF pathology.

\section{Study limitations}

The current study is subject to all limitations inherent to nonrandomized studies. The design was retrospective. We have not accounted for confounders of BNP level other than the degree of congestion. Thus, there may have been other confounders that have not been accounted for and affected mortality like non-cardiac comorbidities, since the study endpoint was allcause mortality during a 6-month follow-up.

\section{Conclusions}

BNP increase at the time of discharge relative to admission is not uncommon and indicates a subset of patients with higher grade of congestion and higher 6-month mortality compared with those who have admission-to-discharge BNP reduction. Mortality is likely related to less efficient decongestion or, more importantly, it may arise from more severe basal clinical compromise. The fact that this group had higher mortality, despite similar BNP levels at admission, suggests that BNP change from admission to discharge is a discriminating factor more important for prognostic assessment compared to absolute BNP measurement on admission. Longitudinal follow-up of BNP on admission and discharge would therefore be a more 
reliable measure for predicting post-discharge mortality.

\section{References}

1. Anand IS, Fisher LD, Chiang YT, Latini R, Masson S, Maggioni AP, Glazer RD, et al. Changes in brain natriuretic peptide and norepinephrine over time and mortality and morbidity in the Valsartan Heart Failure Trial (ValHeFT). Circulation. 2003;107(9):1278-1283.

2. Cohen-Solal A, Logeart D, Huang B, Cai D, Nieminen MS, Mebazaa A. Lowered B-type natriuretic peptide in response to levosimendan or dobutamine treatment is associated with improved survival in patients with severe acutely decompensated heart failure. J Am Coll Cardiol. 2009;53(25):2343-2348.

3. Emdin M, Clerico A, Clemenza F, Galvani M, Latini R, Masson S, Mule P, et al. [Recommendations for the clinical use of cardiac natriuretic peptides]. Ital Heart J Suppl. 2005;6(5):308-325.

4. Drazner MH, Hellkamp AS, Leier CV, Shah MR, Miller LW, Russell SD, Young JB, et al. Value of clinician assessment of hemodynamics in advanced heart failure: the ESCAPE trial. Circ Heart Fail. 2008;1(3):170-177.

5. Gheorghiade M, Follath F, Ponikowski P, Barsuk JH, Blair JE, Cleland JG, Dickstein K, et al. Assessing and grading congestion in acute heart failure: a scientific statement from the acute heart failure committee of the heart failure association of the European Society of Cardiology and endorsed by the European Society of Intensive Care Medicine. Eur J Heart Fail. 2010;12(5):423-433.

6. Goonewardena SN, Gemignani A, Ronan A, Vasaiwala S, Blair J, Brennan JM, Shah DP, et al. Comparison of hand-carried ultrasound assessment of the inferior vena cava and N-terminal pro-brain natriuretic peptide for predicting readmission after hospitalization for acute decompensated heart failure. JACC Cardiovasc Imaging. 2008;1(5):595-601.

7. De Vecchis R, Ciccarelli A, Ariano C. Inferior Vena Cava collapsibility and heart failure signs and symptoms: new insights about possible links. Arq Bras Cardiol. 2012;98(6):544-552 .

8. De Vecchis R, Baldi C. Inferior Vena Cava and Hemodynamic Congestion. Res Cardiovasc Med. 2015;4(3):e28913.

9. O'Connor CM, Abraham WT, Albert NM, Clare R, Gattis Stough W, Gheorghiade M, Greenberg BH, et al. Predictors of mortality after discharge in patients hospitalized with heart failure: an analysis from the Organized Program to Initiate Lifesaving Treatment in Hospitalized Patients with Heart Failure (OPTIMIZE-HF). Am Heart J. 2008;156(4):662-673.

10. Khazanie P, Heizer GM, Hasselblad V, Armstrong PW, Califf RM, Ezekowitz J, Dickstein K, et al. Predictors of clinical outcomes in acute decompensated heart failure: Acute Study of Clinical Effectiveness of Nesiritide in Decompensated Heart Failure outcome models. Am Heart J. 2015;170(2):290-297.

11. McCullough PA. Clinical applications of B-type natriuretic peptide levels in the care of cardiovascular patients. Minerva Cardioangiol. 2004;52(6):479-489.

12. Fonarow GC, Peacock WF, Phillips CO, Givertz MM, Lopatin M. Admission B-type natriuretic peptide levels and in-hospital mortality in acute decompensated heart failure. J Am Coll Cardiol. 2007;49(19):1943-1950.

13. Maisel A, Mueller C, Adams K, Jr., Anker SD, Aspromonte N, Cleland JG, Cohen-Solal A, et al. State of the art: using natriuretic peptide levels in clinical practice. Eur J Heart Fail. 2008;10(9):824-839.

14. De Vecchis R, Esposito C, Cantatrione S. Natriuretic peptide-guided therapy: further research required for stillunresolved issues. Herz. 2013;38(6):618-628.

15. Ponikowski P, Voors AA, Anker SD, Bueno H, Cleland JG, Coats AJ, Falk V, et al. 2016 ESC Guidelines for the diagnosis and treatment of acute and chronic heart failure: The Task Force for the diagnosis and treatment of acute and chronic heart failure of the European Society of Cardiology (ESC). Developed with the special contribution of the Heart Failure Association (HFA) of the ESC. Eur J Heart Fail. 2016;18(8):891-975. 